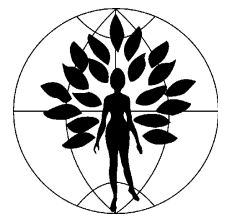

\title{
Contemporary medical therapy for polycystic ovary syndrome
}

\author{
M.S.M. Lanham, D.I. Lebovic, S.E. Domino *
}

Department of Obstetrics and Gynecology, University of Michigan, Ann Arbor, Michigan, USA

Received 19 July 2006; received in revised form 6 August 2006; accepted 9 August 2006

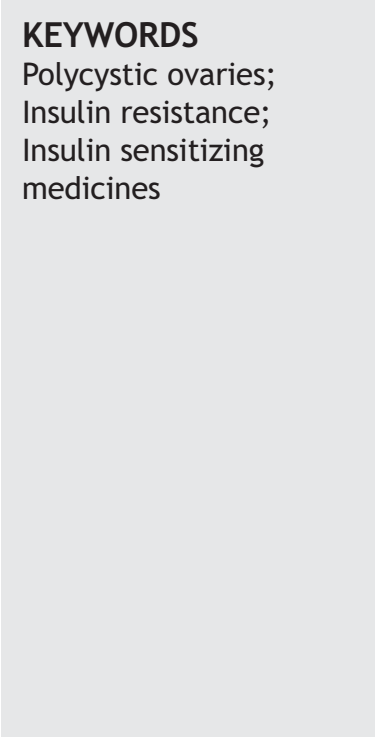

\begin{abstract}
Polycystic ovary syndrome is a multi-system endocrinopathy with longterm metabolic and cardiovascular health consequences. Patients typically present due to symptoms of irregular menstruation, hair growth, or infertility; however, recent management options are aimed at further treating underlying glucose-insulin abnormalities as well as androgen excess for proactive control of symptoms. By a 2003 international consensus conference, diagnosis is made by two out of three criteria: chronic oligoovulation or anovulation after excluding secondary causes, clinical or biochemical evidence of hyperandrogenism (but not necessarily hirsutism due to inter-patient variability in hair follicle sensitivity), and radiological evidence of polycystic ovaries. Traditional medical treatment options include oral contraceptive pills, cyclic progestins, ovulation induction, and anti-androgenic medications (aldosterone antagonist, $5 \alpha$-reductase antagonist, and follicle ornithine decarboxylase inhibitor). Recent pharmacotherapies include insulin-sensitizing medications metformin and two thiazolidinediones (rosiglitazone/Avandia $₫$ and pioglitazone/ Actos $®$ ), a CYP19 aromatase inhibitor (letrozole/Femara $®$ ), and statins to potentially lower testosterone levels.

(c) 2006 International Federation of Gynecology and Obstetrics. Published by Elsevier Ireland Ltd. All rights reserved.
\end{abstract}

\section{Definition}

Perhaps the earliest text on polycystic ovary syndrome (PCOS) was described by Antonio Vallisneri as "...Young

\footnotetext{
* Corresponding author. Department of Obstetrics and Gynecology, Room 6428 Medical Science I Box 0617, 1150 West Medical Center Dr, University of Michigan, Ann Arbor, MI 48109-0617, USA. Tel.: +1 734647 9562; fax: +1 7349368617 .

E-mail address: sedomino@med.umich.edu (S.E. Domino).
}

peasant woman, married, moderately plump, infertile, with ovaries larger than normal, like doves' eggs, lumpy, shiny and whitish" in 1721 [1]. The inherent variability of PCOS was evident even in the initial Irving Stein and Michael Leventhal description in 1935 in their series of patients showing polycystic ovaries, but with other characteristics exhibited non-uniformly, including infertility, hirsutism, and oligomenorrhea [2]. The first international consensus on the clinical criteria for PCOS was held in 2003 by the European

0020-7292/\$ - see front matter ๔ 2006 International Federation of Gynecology and Obstetrics. Published by Elsevier Ireland Ltd. All rights reserved.

doi:10.1016/j.ijgo.2006.08.004 
Society of Human Reproduction and Embryology and the American Society for Reproductive Medicine, at Rotterdam, Netherlands [3]. The consensus definition requires two of the following three criteria, highlighting the range of clinical expressions within the disorder.

1. Polycystic ovaries: 12 or more follicles in an ovary, with each follicle measuring $2-9 \mathrm{~mm}$ in diameter and/or ovarian volume $>10 \mathrm{ml}$. Neither stromal density nor distribution of the follicles is included in this revised definition. One polycystic ovary is sufficient for diagnosis.

2. Oligo-/anovulation: clinically diagnosed as oligo-/ amenorrhea, i.e., menstrual cycles longer than 35 days or less than 10 menstruations per year.

3. Hyperandrogenism: clinical or biochemical.

Note that the criteria for PCOS do not apply to adolescent girls, women using contraceptive steroid pills, or postmenopausal women. The diagnosis of PCOS requires the exclusion of other conditions, including congenital adrenal hyperplasia, non-classic congenital adrenal hyperplasia, Cushing syndrome, acromegaly, androgen-secreting tumors, hyperprolactinemia or prolactinoma, primary hypothyroidism, premature ovarian failure, or drug effects from exogenous androgens. It has been shown that transabdominal and transvaginal ultrasound examinations correlate well; thus, both are acceptable tools for diagnosis, and the choice of which to use can be left to the clinician [4]. In menstruating women, ultrasound examination is most sensitive when performed on the third, fourth, or fifth day of the menstrual cycle.

\section{Prevalence and pathogenesis}

The reported incidence of PCOS ranges from 3\% to $11 \%$ varying by the diagnostic criteria used and the population studied [5]. The prevalence would be expected to be higher with the expanded Rotterdam criteria, as confirmed in one study that demonstrated a prevalence of $12 \%$ [6], and further prevalence studies are needed using the Rotterdam criteria.

\subsection{Evolution and anthropology}

The complexity of PCOS continues to make understanding its pathogenesis a difficult task. Its etiology is only poorly understood, and many theories of pathogenesis have been proposed. It has been hypothesized that during evolutionary history, it may have been advantageous for a woman to be hyperandrogenic and have increased insulin resistance [7]. Insulin resistance may have enhanced a woman's ability to store energy as subcutaneous fat when food was abundant, improving her chances for survival when food was scarce.

\subsection{Genetic and environmental factors}

Heterogeneous phenotypes and lack of a universally accepted definition of PCOS have made genetic studies difficult. There is evidence to support an autosomal dominant genetic model with low penetrance [8]. Genes that have been postulated to be involved in the development of PCOS include the CYP17A gene, which encodes for the P450c $17 \alpha$ enzyme, the CYP11A gene, which encodes for the P450 side-chain cleavage enzyme, the insulin gene, and variable number tandem repeat/VNTR sequences [9]. Mothers of women with PCOS have dyslipidemia, hyperandrogenemia, and serum markers of insulin resistance [10]. Multiple genes seem to be implicated, and polygenic inheritance is likely responsible for the majority of cases.

\subsection{Enhanced serine phosphorylation unification theory}

A theory of pathogenesis that serves as a link between hyperandrogenism and insulin resistance is the enhanced serine phosphorylation unification theory [11]. Serine phosphorylation of the P450c 17 $\alpha$ enzyme enhances its 17,20-lyase activity, leading to increased androgen synthesis within the theca cell. One of the principal mediators of the insulin signaling pathway is insulin receptor substrate-1 (IRS-1). Serine phosphorylation of various sites on IRS-1 leads to decreased activity of the mediator when insulin signaling occurs, blunting the normal transport of glucose into the cell. The body responds to this relative hyperglycemia with hyperinsulinemia.

Insulin stimulates androgen synthesis in both the ovaries and in the adrenal glands [12]. Insulin acts synergistically with luteinizing hormone and augments steroid synthesis in the ovaries, which leads to follicle arrest and anovulation [13]. Insulin also inhibits the production of sex hormone binding globulin in the liver leading to higher levels of serum-free testosterone.

\section{Treatment}

\subsection{Non-pharmacologic treatment}

The diversity of the clinical presentation and pathophysiology of PCOS is mirrored by the numerous treatment options. The various treatments can 
be categorized among those that control cutaneous manifestations of hyperandrogenemia, those that restore regular ovulation and menstruation, and those that affect insulin resistance. Treatments in one of these categories are not limited to effects only in its own category [14].

Weight loss of only $5-7 \%$ has been shown to improve manifestations of hyperandrogenism, decrease hyperinsulinemia, and restore ovulation and fertility in up to $75 \%$ of women [15]. There is no evidence that any particular diet regimen is more beneficial than another for PCOS women.

Utilizing ovarian drilling or diathermy, ovulation can be restored in $80 \%$ of women, and the pregnancy rate is $50 \%$ in women who are treated with surgery [16]. Laparoscopic ovarian diathermy has been shown to be safe and cost-effective for treating clomiphene-resistant PCOS [17].

\subsection{Treatment for acne and hirsutism}

Treatments for acne and hirsutism in PCOS individuals strive to decrease serum androgen levels, by lowering androgen production or by increasing androgen binding to plasma-binding proteins, and to block androgen activity in the target tissue [14]. Contraceptive steroid pills, specifically estrogenprogestin combination therapy, are used by many clinicians as the first-line treatment for acne and hirsutism in individuals with PCOS who do not desire immediate fertility. The estrogen suppresses luteinizing hormone release from the pituitary, which suppresses ovarian androgen production [14]. Estrogen also increases hepatic production of sex hormone-binding globulin, decreasing the unbound serum testosterone. Though oral contraceptive pills will improve acne, hirsutism, and protect against endometrial hyperplasia due to unopposed estrogen, this method of treatment does not positively affect insulin resistance $[18,19]$.

In patients in whom treatment with oral contraceptive pills may be contraindicated (e.g., smokers above age 35 , individuals with a history of thrombotic disease), progestins may be given monthly or every 2 3 months to prevent endometrial hyperplasia. This treatment will not affect hirsutism, acne, or improve insulin resistance.

Antiandrogen treatments are also used to combat acne and hirsutism. Used predominantly in Canada, Mexico, and Europe, cyproterone acetate competitively inhibits the binding of testosterone and $5 \alpha$ dihydrotestosterone to the androgen receptor [20] and effectively treats hirsutism and acne. Antiandrogen treatments that are utilized in the United States include spironolactone and finasteride. Drospirenone is an analogue of spironolactone with some anti- mineralocorticoid and antiandrogenic activity; it is available in combination with estradiol in the oral contraceptive pill Yasmin $₫$ or Yaz $₫$ (Berlex Laboratories Inc., Monteville, NJ 07045 USA) [21]. When used in higher doses $(\sim 100-200 \mathrm{mg} /$ day $)$, spironolactone has moderate antiandrogenic effects. It inhibits steroidogenic enzymes and binds the dihydrotestosterone receptor at the hair follicle [22]. Side effects include irregular uterine bleeding. Contraception is required during administration of spironolactone (pregnancy category D). Use of spironolactone during pregnancy is contraindicated, as it leads to incomplete virilization of male fetuses. As the drug has been found to have synergistic effects when combined with oral contraceptive pills, these two are often prescribed simultaneously [14].

Flutamide, a nonsteroidal antiandrogen, is effective in the treatment of hirsutism, but concern about the side effect of liver dysfunction has blunted its widespread use [23]. Finasteride is a competitive inhibitor of the enzyme type $25 \alpha$-reductase, and systemic use has been shown to decrease circulating dihydrotestosterone and to treat hirsutism [24]. Topical use is not effective since type $15 \alpha$-reductase is present in the pilosebaceous unit [14]. Because the use of finasteride during the first trimester increases risks of hypospadias in male fetuses, contraception is also mandatory with this drug (pregnancy category $\mathrm{X}$ ).

Eflornithine hydrochloride (Vaniqa ${ }^{\circledR}$; SkinMedica Inc., Carlsbad, CA 92008, USA) is a topical medication approved for use in treating facial hirsutism. It inhibits ornithine decarboxylase, a critical enzyme in initiating cell division, and thus, it acts in the follicle to disrupt hair growth [25]. It requires 4-8 weeks of treatment for results and is a pregnancy category $C$ drug. Clinical data in PCOS women is limited.

The above therapies will all target hair in the long term. They may take as long as 6-9 months to have maximal effects, and discontinuation of each drug is associated with recurrence of hirsutism. In the short term, patients should be counseled to pursue more direct methods of removing hair. Laser hair removal delivers thermal injury to the follicle by targeting the follicular melanin [26]. It works best in individuals with light skin and dark hair, but it may cause pigment changes in the skin. It should be avoided in patients who tend to form keloids or hypertrophic scars.

\subsection{Drug treatment to restore ovulation/ fertility}

Lowering insulin levels by improving insulin sensitivity improves ovulation, restores menstruation, and improves fertility, as well as symptoms of hyperandrogenemia $[27,28]$. Thus, insulin-sensitizing agents are subjects of continued research on improving 
ovulation, as well as improving the cardiovascular risk factors associated with hyperinsulinemia, in PCOS women. The research supporting these drugs will be presented in the next section.

Ovulation rates up to $80 \%$ contrast with pregnancy rates of $30-40 \%$ in cycles with or without clomiphene citrate (CC) in PCOS women [29]. Adding metformin increases ovulation rate and conception rate compared to CC alone [30]. Newer ovulation induction agents include the aromatase inhibitors such as letrozole (Femara $\AA$; Novartis Pharmaceutical Corp., East Hanover, NJ 07936 USA). Letrozole is a nonsteroidal inhibitor that binds reversibly to the heme group of aromatase CYP19. Its primary use is for adjuvant treatment of postmenopausal women with hormone receptor positive early breast cancer (pregnancy category $\mathrm{D}$ ). In the setting of PCOS, by decreasing estrogen production, it leads to an increase in follicle stimulating hormone (FSH) release by the pituitary, without the ill-toward anti-estrogenic properties of CC. Increased FSH then leads to follicular development and eventual ovulation. Compared with CC, letrozole may (1) decrease the number of mature follicles, (2) decrease the risk of ovarian hyperstimulation syndrome, and (3) increase the rates of both ovulation and pregnancy [31]. In a randomized trial in CC-resistant PCOS women on metformin, letrozole led to a higher delivery rate than continued CC therapy [32]. Side effects include hot flashes, nausea, hair thinning, arthralgia, myalgia, and arthritis, although side effects have not been shown to affect compliance, a problem seen more frequently in CC recipients.

\subsection{Therapies that affect insulin resistance}

The French lilac or Goat's rue plant (Galega officianlis) was used to treat diabetes mellitus in medieval times [33]. It was determined that the plant contained guanidine, which had anti-hyperglycemic properties, and is structurally related to biguanides, one of which is metformin. Metformin was marketed in Europe starting in 1979, and it was approved for use by the United States in 1995. Due to the history of lactic acidosis in this class, it is avoided in individuals with kidney or liver disease, in situations that increase the risk for lactic acidosis, and before or after procedures when patients will receive an intravenous dye load.

Metformin is widely used today in patients with type II diabetes mellitus and with PCOS, but the exact mechanism by which it functions remains a mystery. What is known is that it both enhances peripheral uptake and inhibits hepatic glucose production; thus, it is an anti-hyperglycemic rather than a hypoglycemic agent [34]. Significant weight reduction has been seen with metformin therapy [35]. Its side effects include nausea, diarrhea, abdominal discomfort, metallic taste, and anorexia, which can occur in up to $20 \%$ of patients. Side effects can be reduced by taking the drug with meals and by increasing the dose slowly. It is pregnancy class $B$, and one study has not found an adverse affect on growth or motor-social development in the first 18 months of life [36].

Metformin has been shown to be effective in ovulation induction as the sole agent and in conjunction with CC $[30,37]$. The presence of insulin resistance is a positive predictor of metformin response in PCOS women [38]. Long-term therapy with metformin decreases progression from insulin resistance to frank diabetes mellitus in PCOS women, but short-term therapy of as little as 2 weeks helps to improve ovulation and fertility $[39,40]$.

Three thiazolidinediones have been used in clinical practice: troglitazone (Rezulin $®$; Parke-Davis/Warner-Lambert, now Pfizer, Inc., New York, NY 10017,

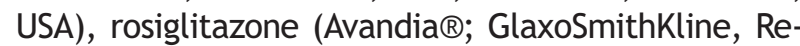
search Park Triangle, NC 27709, USA), and pioglitazone (Actos $®$; Tekada Pharmaceuticals America, Inc., Lincolnshire, IL 60069, USA, and Eli Lilly and Company, Indianapolis, IN 46285, USA). Troglitazone was withdrawn from the market due to hepatic toxicity in 2000. The thiazolidinediones work as selective agonists for nuclear peroxisome proliferator-activated receptor- $\gamma$ (PPAR- $\gamma$ ). Binding to PPAR- $\gamma$ activates genes that regulate carbohydrate and lipid metabolism, and the drugs' principal effects are to increase insulin sensitivity in peripheral tissue and lower glucose production by the liver [41]. Because of the history of this class of drugs, liver enzymes are generally monitored during therapy with thiazolidinediones. Metabolism occurs in the liver; rosiglitazone by CYP2C8, pioglitazone by both CYP3A4 and CYP2C8.

Studies with troglitazone in PCOS women showed improvements in insulin resistance, hyperandrogenemia, and glucose tolerance [42]. Ovulation was significantly increased, free testosterone levels decreased, and levels of sex hormone-binding globulin increased. Though troglitazone is no longer available, studies with rosiglitazone and pioglitazone have demonstrated similar results $[43,44]$. The drugs are pregnancy class $\mathrm{C}$. Because they work by different mechanisms, thiazolidinediones and metformin may be used together in type II diabetes mellitus. Further research will need to be done to determine the effects of this combination in individuals with PCOS.

\subsection{Statin medications}

Statins are a novel therapeutic approach to PCOS and have shown promise in early in vitro studies as well as a single prospective, randomized trial. 
Mevastatin-treated cultured ovarian theca-interstitial cells had diminished proliferation and steroidogenesis [45]. A clinical trial compared simvastatin plus oral contraceptive pills to an oral contraceptive group alone [46]. The statin group showed significantly lowered testosterone levels and normalized gonadotropin levels; however, there was no significant difference between the groups on the Ferriman-Gallway scale, which is used to quantify the severity of a patient's hirsutism.

\section{Future directions and research}

The realization that PCOS is intimately linked to insulin resistance and the host of metabolic problems associated with type II diabetes mellitus, especially the increased risk of cardiovascular disease, has led to a shift in paradigm in the treatment of this syndrome. Traditional treatment focused on reproductive and aesthetic issues. PCOS is now recognized as a multisystem syndrome with long-term health consequences. Induction of ovulation and control of hyperandrogenic symptoms has been augmented by the use of insulin-sensitizing drugs.

The controversies of the expansion of the Rotterdam criteria will require research into those women who were not previously included as PCOS patients to determine if treating them with the same drugs and protocols is safe and advantageous. Further research into the enhanced serine phosphorylation theory may lead to drugs that target these phosphorylation sites.

While studies have shown combined therapy with metformin and CC to be superior to CC alone in PCOS women and letrozole to be superior to $\mathrm{CC}$ alone in PCOS women, there are no studies that look at the effects of combining metformin with letrozole. Such a protocol may give patients the long-term benefits of controlling insulin levels along with the shortterm benefits of increased FSH secretion and regular ovulation and menstruation.

\section{References}

[1] Battaglia C. The role of ultrasound and Doppler analysis in the diagnosis of polycystic ovary syndrome. Ultrasound Obstet Gynecol 2003;22:225-32.

[2] Stein I, Leventhal M. Amenorrhea associated with bilateral polycystic ovaries. Am J Obstet Gynecol 1935;29:181-91.

[3] The Rotterdam ESHRE/ASRM-Sponsored PCOS Consensus Workshop group. Revised 2003 consensus on diagnostic criteria and long-term health risks related to polycystic ovary syndrome (PCOS). Hum Reprod 2004;19:41-7.

[4] Farquhar CM, Birdsall M, Manning P, Mitchell JM, France JT. The prevalence of polycystic ovaries on ultrasound scanning in a population of randomly selected women. Aust $\mathrm{N} \mathrm{Z} \mathrm{J}$ Obstet Gynaecol 1994;34:67-72.
[5] Knochenhauer ES, Key TJ, Kahsar-Miller M, Waggoner W, Boots LR, Azziz R. Prevalence of the polycystic ovary syndrome in unselected black and white women of the southeastern United States: a prospective study. J Clin Endocrinol Metab 1998;83:3078-82.

[6] Lowe P, Kovacs G, Howlett D. Incidence of polycystic ovaries and polycystic ovary syndrome amongst women in Melbourne, Australia. Aust N Z J Obstet Gynaecol 2005;45:17-9.

[7] Balen AH. Polycystic ovary syndrome: a guide to clinical management. London: Taylor \& Francis; 2005. 210 pp.

[8] Legro RS, Spielman R, Urbanek M, Driscoll D, Strauss III JF, Dunaif A. Phenotype and genotype in polycystic ovary syndrome. Recent Prog Horm Res 1998;53:217-56.

[9] Franks S, Gharani N, McCarthy M. Candidate genes in polycystic ovary syndrome. Hum Reprod Update 2001;7:405-10.

[10] Sam S, Legro RS, Essah PA, Apridonidze T, Dunaif A. Evidence for metabolic and reproductive phenotypes in mothers of women with polycystic ovary syndrome. Proc Natl Acad Sci U S A 2006;103:7030-5.

[11] Dunaif A, Xia J, Book CB, Schenker E, Tang Z. Excessive insulin receptor serine phosphorylation in cultured fibroblasts and in skeletal muscle. A potential mechanism for insulin resistance in the polycystic ovary syndrome. J Clin Invest 1995;96:801-10.

[12] Arslanian SA, Lewy V, Danadian K, Saad R. Metformin therapy in obese adolescents with polycystic ovary syndrome and impaired glucose tolerance: amelioration of exaggerated adrenal response to adrenocorticotropin with reduction of insulinemia/insulin resistance. J Clin Endocrinol Metab 2002;87:1555-9.

[13] Willis DS, Watson H, Mason HD, Galea R, Brincat M, Franks S. Premature response to luteinizing hormone of granulosa cells from anovulatory women with polycystic ovary syndrome: relevance to mechanism of anovulation. J Clin Endocrinol Metab 1998;83:3984-91.

[14] Ehrmann DA. Polycystic ovary syndrome. N Engl J Med 2005;352:1223-36.

[15] Kiddy DS, Hamilton-Fairley D, Bush A, Short F, Anyaoku V, Reed $\mathrm{MJ}$, et al. Improvement in endocrine and ovarian function during dietary treatment of obese women with polycystic ovary syndrome. Clin Endocrinol (Oxf) 1992;36:105-11.

[16] ACOG Committee on Practice Bulletins-Gynecology. Clinical Management Guidelines for Obstetrician-Gynecologists Number 34, February 2002. Management of infertility caused by ovulatory dysfunction. Obstet Gynecol 2002;99:347-58.

[17] Farquhar CM, Williamson K, Brown PM, Garland J. An economic evaluation of laparoscopic ovarian diathermy versus gonadotrophin therapy for women with clomiphene citrate resistant polycystic ovary syndrome. Hum Reprod 2004;19:1110-5.

[18] Diamanti-Kandarakis E, Baillargeon JP, luorno MJ, Jakubowicz DJ, Nestler JE. A modern medical quandary: polycystic ovary syndrome, insulin resistance, and oral contraceptive pills. J Clin Endocrinol Metab 2003;88:1927-32.

[19] Vrbikova J, Cibula D. Combined oral contraceptives in the treatment of polycystic ovary syndrome. Hum Reprod Update 2005;11:277-91.

[20] Golland IM, Elstein ME. Results of an open one-year study with Diane-35 in women with polycystic ovarian syndrome. Ann N Y Acad Sci 1993;687:263-71.

[21] Batukan C, Muderris II. Efficacy of a new oral contraceptive containing drospirenone and ethinyl estradiol in the longterm treatment of hirsutism. Fertil Steril 2006;85:436-40.

[22] Young RL, Goldzieher JW, Elkind-Hirsch K. The endocrine effects of spironolactone used as an antiandrogen. Fertil Steril 1987;48:223-8.

[23] Muderris II, Bayram F, Guven M. Treatment of hirsutism with lowest-dose flutamide $(62.5 \mathrm{mg} /$ day). Gynecol Endocrinol 2000;14:38-41. 
[24] Falsetti L, Gambera A, Legrenzi L, lacobello C, Bugari G. Comparison of finasteride versus flutamide in the treatment of hirsutism. Eur J Endocrinol 1999;141:361-7.

[25] Balfour JA, McClellan K. Topical eflornithine. Am J Clin Dermatol 2001;2:197-201 [discussion 2].

[26] Hobbs L, Ort R, Dover J. Synopsis of laser assisted hair removal systems. Skin Therapy Lett 2000;5:1-5.

[27] Moran LJ, Noakes M, Clifton PM, Tomlinson L, Galletly C, Norman RJ. Dietary composition in restoring reproductive and metabolic physiology in overweight women with polycystic ovary syndrome. J Clin Endocrinol Metab 2003;88:812-9.

[28] Nestler JE, Jakubowicz DJ, Evans WS, Pasquali R. Effects of metformin on spontaneous and clomiphene-induced ovulation in the polycystic ovary syndrome. N Engl J Med 1998;338:1876-80.

[29] Kousta E, White DM, Franks S. Modern use of clomiphene citrate in induction of ovulation. Hum Reprod Update 1997;3:359-65.

[30] Vandermolen DT, Ratts VS, Evans WS, Stovall DW, Kauma SW, Nestler JE. Metformin increases the ovulatory rate and pregnancy rate from clomiphene citrate in patients with polycystic ovary syndrome who are resistant to clomiphene citrate alone. Fertil Steril 2001;75:310-5.

[31] Casper RF, Mitwally MF. Review: aromatase inhibitors for ovulation induction. J Clin Endocrinol Metab 2006;91:760-71.

[32] Sohrabvand F, Ansari S, Bagheri M. Efficacy of combined metformin-letrozole in comparison with metformin-clomiphene citrate in clomiphene-resistant infertile women with polycystic ovarian disease. Hum Reprod 2006;21:1432-5.

[33] Witters LA. The blooming of the French lilac. J Clin Invest 2001;108:1105-7.

[34] Stumvoll M, Nurjhan N, Perriello G, Dailey G, Gerich JE. Metabolic effects of metformin in non-insulin-dependent diabetes mellitus. N Engl J Med 1995;333:550-4.

[35] Harborne LR, Sattar N, Norman JE, Fleming R. Metformin and weight loss in obese women with polycystic ovary syndrome: comparison of doses. J Clin Endocrinol Metab 2005;90:4593-8.

[36] Glueck CJ, Goldenberg N, Pranikoff J, Loftspring M, Sieve L, Wang P. Height, weight, and motor-social development during the first 18 months of life in 126 infants born to 109 mothers with polycystic ovary syndrome who conceived on and continued metformin through pregnancy. Hum Reprod 2004;19:1323-30.
[37] Lord JM, Flight IH, Norman RJ. Insulin-sensitising drugs (metformin, troglitazone, rosiglitazone, pioglitazone, D-chiroinositol) for polycystic ovary syndrome. Cochrane Database Syst Rev 2003:CD003053.

[38] Eisenhardt S, Schwarzmann N, Henschel V, Germeyer A, von Wolff M, Hamann A, et al. Early effects of metformin in women with polycystic ovary syndrome: a prospective randomized, double-blind, placebo-controlled trial. J Clin Endocrinol Metab 2006;91:946-52.

[39] Jakubowicz DJ, luorno MJ, Jakubowicz S, Roberts KA, Nestler JE. Effects of metformin on early pregnancy loss in the polycystic ovary syndrome. J Clin Endocrinol Metab 2002;87:524-49.

[40] Khorram O, Helliwell JP, Katz S, Bonpane CM, Jaramillo L. Two weeks of metformin improves clomiphene citrateinduced ovulation and metabolic profiles in women with polycystic ovary syndrome. Fertil Steril 2006;85:1448-51.

[41] Davis S. Insulin, Oral Hypoglycemic Agents, and the Pharmacology of the Endocrine Pancreas. In: Goodman LS, Gilman A, Brunton LL, Lazo JS, Parker KL, editors. Goodman \& Gilman's the pharmacological basis of therapeutics. New York: McGraw-Hill; 2006. p. 1613-46.

[42] Azziz R, Ehrmann D, Legro RS, Whitcomb RW, Hanley R, Fereshetian AG, et al. Troglitazone improves ovulation and hirsutism in the polycystic ovary syndrome: a multicenter, double blind, placebo-controlled trial. J Clin Endocrinol Metab 2001;86:1626-32.

[43] Ghazeeri G, Kutteh WH, Bryer-Ash M, Haas D, Ke RW. Effect of rosiglitazone on spontaneous and clomiphene citrateinduced ovulation in women with polycystic ovary syndrome. Fertil Steril 2003;79:562-6.

[44] Rouzi AA, Ardawi MS. A randomized controlled trial of the efficacy of rosiglitazone and clomiphene citrate versus metformin and clomiphene citrate in women with clomiphene citrate-resistant polycystic ovary syndrome. Fertil Steril 2006;85:428-35.

[45] Izquierdo D, Foyouzi N, Kwintkiewicz J, Duleba AJ. Mevastatin inhibits ovarian theca-interstitial cell proliferation and steroidogenesis. Fertil Steril 2004;82(Suppl 3):1193-7.

[46] Duleba AJ, Banaszewska B, Spaczynski RZ, Pawelczyk L. Simvastatin improves biochemical parameters in women with polycystic ovary syndrome: results of a prospective, randomized trial. Fertil Steril 2006;85:996-1001. 